University of Nebraska - Lincoln

DigitalCommons@University of Nebraska - Lincoln

Congreso internacional sobre iconografía

precolombina, Barcelona 2019. Actas.

$7-20-2020$

\title{
Los textiles mayas contemporáneos de Yucatán (México) en el espejo de la iconografía precolombina
}

Danielle Dupiech Cavaleri

Follow this and additional works at: https://digitalcommons.unl.edu/actas2019

Part of the American Material Culture Commons, Indigenous Studies Commons, Museum Studies Commons, Other History of Art, Architecture, and Archaeology Commons, and the Other Languages, Societies, and Cultures Commons

This Article is brought to you for free and open access by the Zea E-Books at DigitalCommons@University of Nebraska - Lincoln. It has been accepted for inclusion in Congreso internacional sobre iconografía precolombina, Barcelona 2019. Actas. by an authorized administrator of DigitalCommons@University of Nebraska - Lincoln. 


\title{
Los textiles mayas contemporáneos de Yucatán (México) en el espejo de la iconografía precolombina
}

\author{
Danielle Dupiech Cavaleri \\ Doctora en antropología social de la E.H.E.S.S. (Paris, Francia)
}

\begin{abstract}
Resumen
La investigación llevada con unas veinte bordadoras de 50 a 105 años de edad en los pueblos de Maní, Xaya, Xócen y Xohuayan ha permitido rescatar la información contenida en los dechados que las mujeres conservan preciosamente.

Los datos recopilados que fueron analizados, comparándolos con elementos encontrados en los informes publicados sobre excavaciones arqueológicas; con la iconografía presente en la cerámica prehispánica; con los motivos de los atavíos de los personajes que aparecen en las estelas, los bajos relieves, las estatuas de barro y las pinturas murales; con los pictogramas encontrados en los códices Mayas; con los mitos y leyendas evocados en los textos de la literatura tal como a los mitos y leyendas contemporáneos y por fin, con el análisis semántico, atestan de la continuidad de la cosmogonía maya vinculada a través de los textiles.
\end{abstract}

Palabra clave: bordado, símbolo, simbología, cosmogonía

\begin{abstract}
Contemporary Mayan textiles of Yucatán in the mirror of prehispanic iconography. Research results from about twenty embroiderers ranging in age from 50-105 years old in the villages of Mani, Xaya, Xocen and Xohuayan has allowed the safeguarding of information in samplers (dechados) these women carefully guarded and cherished.

The collected data was analyzed and compared with archaeological excavation reports; the iconography present in prehispanic ceramics; the motifs present in the clothing of characters in stelae, bas-reliefs, clay statuettes and frescoes, pictograms from the Mayas codices, elements in the myths and legends of the great Mayan literary texts, and finally a semantic analysis lays witness to the continuity of textiles as a part of Mayan cosmogony.
\end{abstract}

Keywords: embroidery, symbol, symbolism, cosmogony

\begin{abstract}
ช్ํำ
凹ْ

Pocos estudios han profundizado sobre el contenido cultural de los textiles mayas de Yucatán. Esta falta de interés tiene que ver con la historia de la Península. Cuando llegaron los españoles descubrieron que crecía un algodón de alta calidad y empezaron a explotarlo. Obligaron a las tejedoras mayas a sustituir el tejido de sus telas brocadas por telas lisas como parte del tributo que los indígenas debían pagar a los encomenderos, a cambio de su protección y de su acción civilizadora (de la Garza, 1983: Tomo I, 97). A partir de la segunda mitad del siglo XVI, los indígenas nobles que usaban ropas ricamente brocadas, los dejaron para adoptar la moda europea (Quezada, 2010: 51). Quizá por estas razones la información relativa a la descripción de los textiles mayas de la Península en las crónicas fue muy escasa.

Me fije por casualidad que los textiles contemporáneos bordados por las mujeres yucatecas sobre telas industriales podrían tener un sentido y tome la decisión de investigarlos. Del año 2009 al año 2014, observe los rituales y las fiestas patronales y entreviste a veinte y cuatro bordadoras de 50 a 105 años en los pueblos de Maní, Xáya, Xocén y Xohuayan (mapa1) que tenían plasmada la memoria de sus motivos en sus dechados.

Un dechado (Foto 1) es un trozo de tela industrial blanco, de tamaño variable (no mas de 80 centímetros de largo y 40 centímetros de ancho) sobre el que han sido guardados los motivos que las mujeres reproducen en los hipiles, manteles, servilletas, sudarios o estolas. Por lo general, los motivos son de pequeñas dimensiones,
\end{abstract}




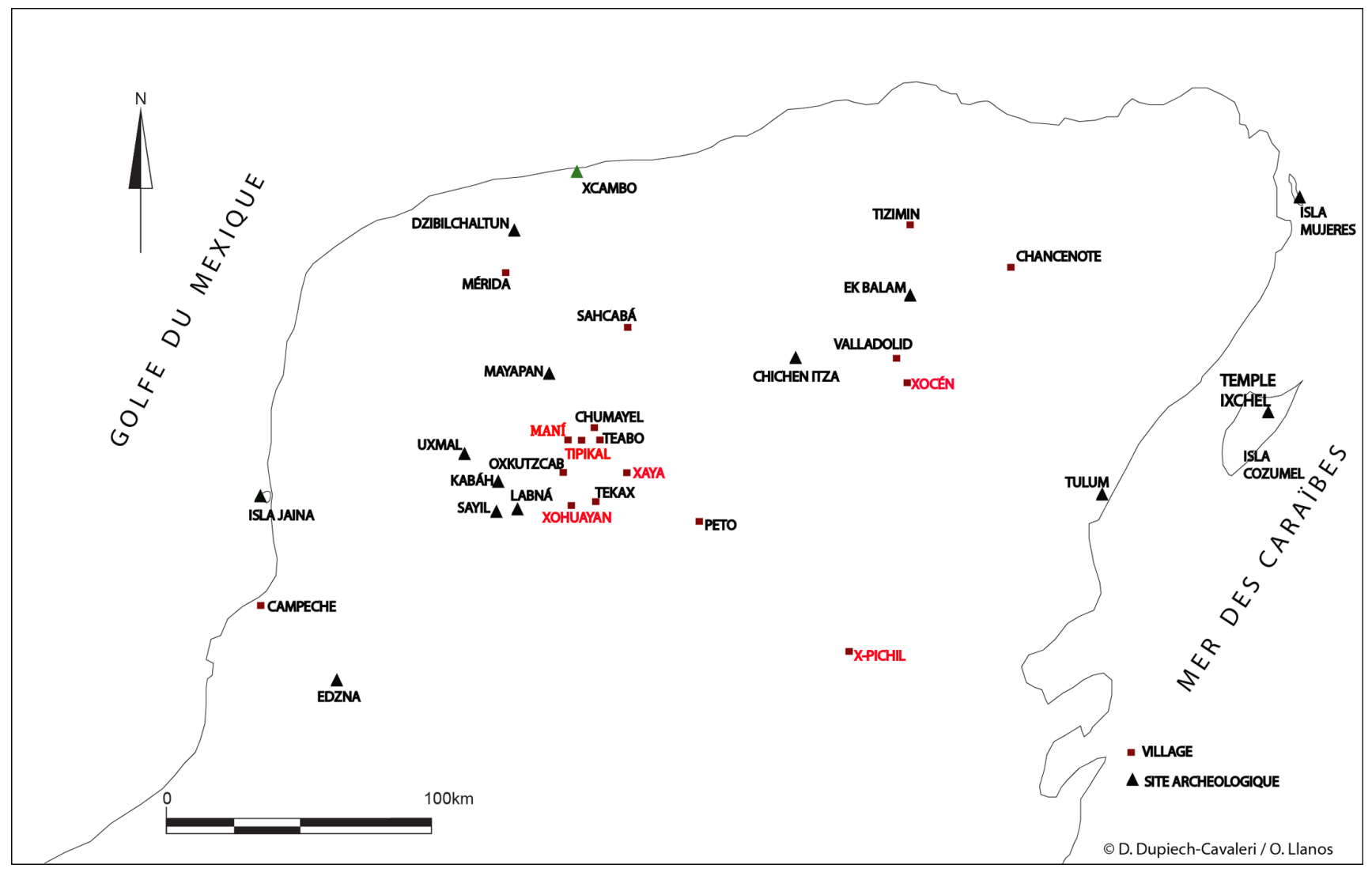

Mapa 1: Península de Yucatán (México).

de apenas unos centímetros cuadrados. Están bordados sobre pequeños trozos de tela de algodón o de cañamazo que pueden llevar uno o varios dibujos. Las mujeres juntan estos pedazos para coserlos en un soporte más grande de algodón para no extraviarlos. Las bordadoras amplifican los motivos cuando los reproducen. En el pasado, a los dechados se les otorgaba una gran importancia pero ahora no le interesan a nadie pues estos motivos ya no se utilizan. Algunas bordadoras dicen que "cuando no tienen herencia sus dechados, se queman... no se pueden transformar en trapo porque nadie lo puede pisar". ¿Por qué? ¡ “Porque lleva el k'in'am de la persona!”. Este universo simbólico pertenece a la cosmogonía de los mayas de Yucatán plasmado en los textiles. Las jóvenes bordadoras prefieren los dibujos de flores copiados en libros vendidos en las tiendas donde compran sus hilos.

\section{Uso de los textiles en los rituales.}

Si bien existía la costumbre de vestir a los santos en España, Diego de Landa en la Relación de las Cosas de Yucatán (2003: 89) refiere que, en 1517, Francisco Hernández que llegaba de Cuba, bautizó con el nombre de Isla
Mujeres a la isla donde desembarcó «por los ídolos que allí halló de las diosas de aquella tierra como Aixchel, Ixchelbeliax, Ixbunic, Ixbunieta, y que estaban vestidas de la cintura abajo y cubiertos los pechos como usan las indias». Los Mayas de Yucatán ofrecen todavía textiles bordados que llaman $k^{\prime} e x$ a sus vírgenes, estolas a las cruces, sudarios a los cristos, manteles a los altares, servilletas ceremoniales para envolver o presentar las ofrendas durante el hanal pixan o algunos rituales agrícolas y para envolver velas durante las procesiones (foto 2). K'ex significa intercambio/permutación/cambio. K'ex significa también algo que se debe de hacer. Durante el Hanal pixan se ponen manteles con motivos y colores especiales sobre los altares en las casas y se depositan tortillas sobre servilletas. Hanal pixan (literalmente comida para los espíritus) marca la temporada de los días que se festejan en las casas el 30 y 31 de octubre para acoger a los pixanes que regresan cada año para visitar a los vivos y permanecen todo el mes de noviembre. Estos días coinciden con los días del calendario maya de la cosecha del maíz nuevo y se hace una ofrenda de elote al altar. Existe también la costumbre de cambiar los manteles blancos en los cuales guardan los huesos de sus difuntos en los cementerios. 

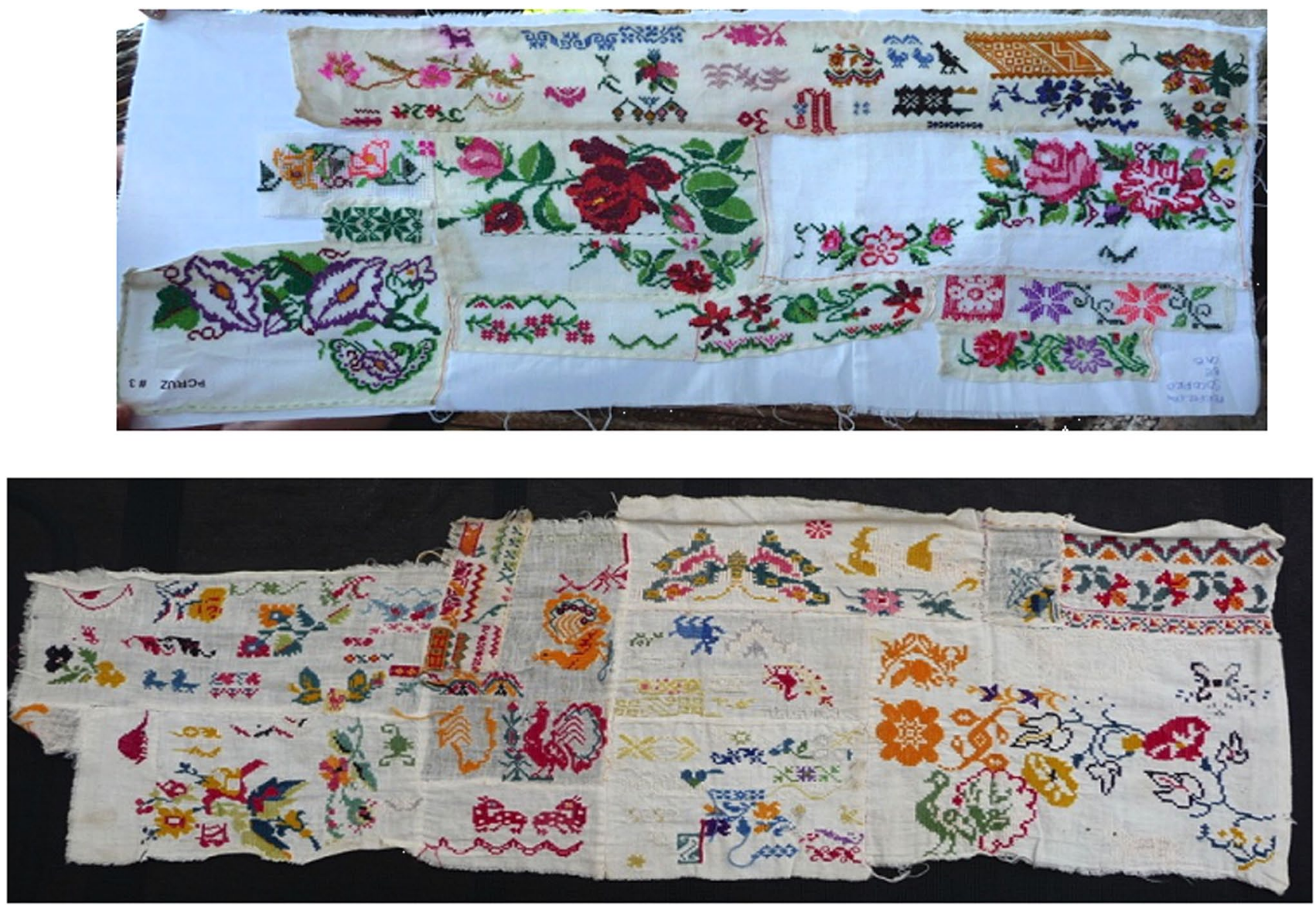

Foto 1: Dechados de Perfecta Socorro Be Cab (Maní) y Carlota Dzul (Xaya), Yucatán, México. (C) D. Dupiech Cavaleri.

La información encontrada en el contexto arqueológico atestigua el papel importante de los textiles. Se menciona textiles asociados con cuchillos sacrificiales en el cenote de Chichén Itzá (Mahler Lothrop, 1992: 75). Si bien la mayoría de los textiles encontrados en este cenote provenían de las ropas de las personas sacrificadas, los arqueólogos también exhumaron trozos de tela gruesa, cuya técnica parece asemejarse a la de los fragmentos de textiles descubiertos durante las excavaciones de la Cueva de Barton Creek en Belice (Mexicon: June 2004, Vol. XXVI, 52). Estos últimos habían sido utilizados para envolver paquetes de ofrendas que contenían almendras, semillas de chile, de cacao, copal, nance/chi y más particularmente mazorcas de maíz. Todas estas plantas envueltas en textiles se situaban en un contexto de ofrendas dedicadas a una divinidad de la tierra y se interpretan como un pago simbólico después de una cosecha, en el que el textil formaba parte integrante de la ofrenda (Mexicon, June 2004: Vol. XXVI, 54).

El análisis de Los códices mayas (1985) ofrece claves para entender el papel del textil en las ceremonias y rituales contemporáneos. Su presencia durante los rituales es poco relevante en el códice de Dresde, pero se manifiesta en el códice Trocortesiano o códice de Madrid.

La interpretación de los glifos que acompañan los pictogramas del Códice de Dresde, realizada por Linda Schele y Nikolai Gruber (1997) para estudiar los calendarios mayas, contribuyó a que entendiera el papel que juegan los textiles en algunos augurios. Las representaciones de textiles relacionados con ofrendas en el Códice Dresde aparecen solo en dos ocasiones: de manera muy clara en la lámina 2 y en forma más sutil en la lámina $22 b$ (foto 3). La lámina 2 se compone de 4 pictogramas: $a, b$, $c$ y $d$. En cada pictograma aparece el textil. En el pictograma $b$ se nota la presencia del Dios de la muerte sosteniendo con una mano un marco donde va tendida una tela lisa de color blanco, la que perfora con una aguja enhebrada sostenida con la otra mano. En el pictograma 2 $c$, observamos a dos personajes. El primer representa al Dios del Maíz que sostiene un hilo con una mano y con la otra una aguja. A la derecha se aprecia una divinidad que Schele y Gruber identificaron como Itzamna 

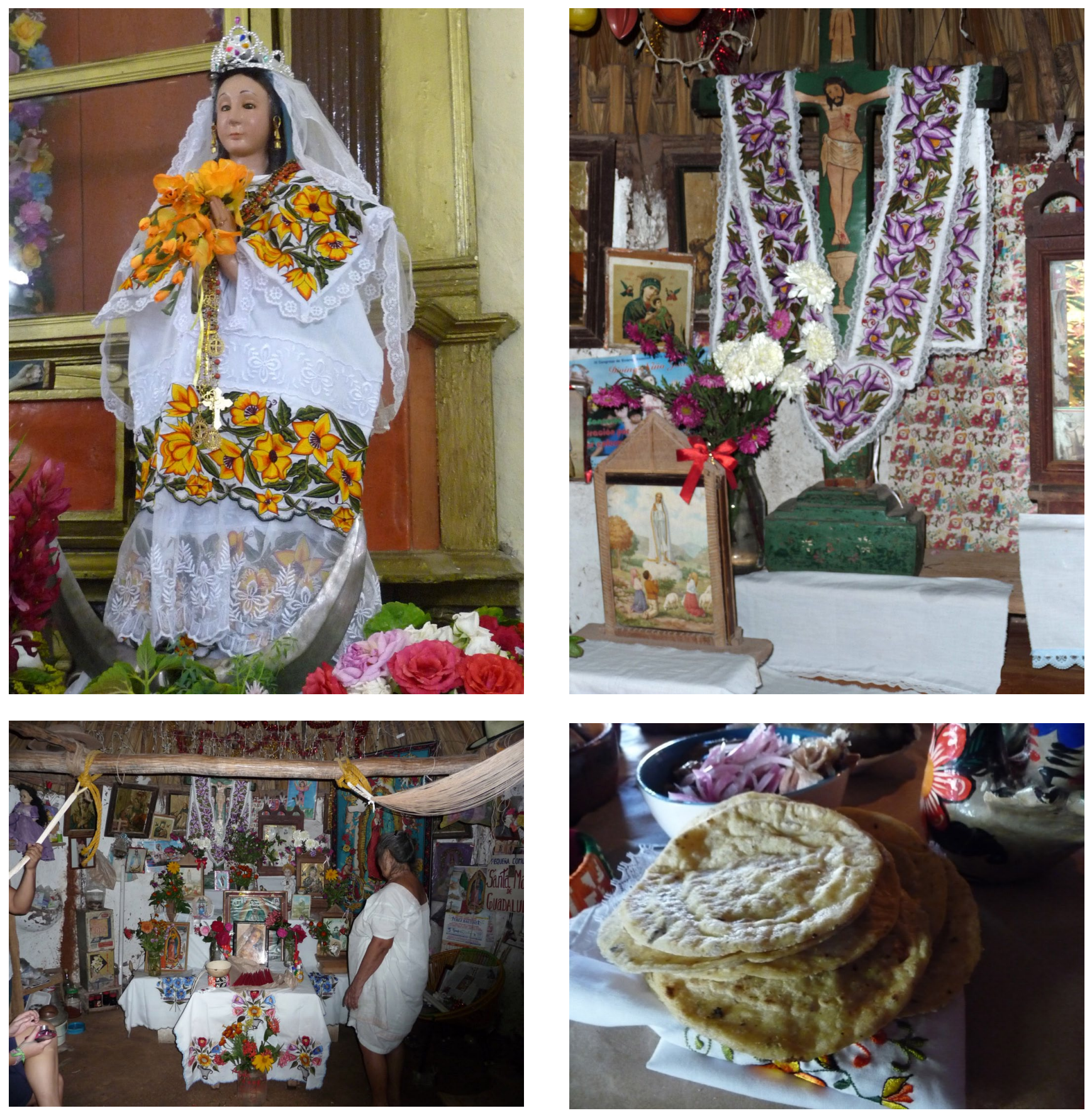

Foto 2: textiles a las vírgenes y a las cruces, manteles de colores especiales sobre los altares y servilletas ceremoniales para depositar ofrendas de tortillas (C) D. Dupiech Cavaleri.

bajo su apariencia de Dios Viejo. Este personaje sostiene un marco con la mano derecha y con otra algo que parecería ser una aguja. Las divinidades estarían apretándose para producir un textil relacionado con la cosecha del maíz para ofrecerlo. Finalmente, el último dibujo nos muestra la Diosa de la Luna, Sak Sik, conocida también bajo el nombre de Ixchel, sosteniendo una ofrenda en sus manos. Según Schele y Gruber (1997: 37), el texto glíptico que aparece arriba indica: "ella recibe el huipil o enagua ( $u$ k'am u pik). El Dios de la Muerte que aparece a su lado, presenta un textil identificado como una enagua. En la lámina 22, pictograma $b$ (foto 3 ), podemos observar también a tres divinidades, cada una sostiene el glifo del maíz en su mano derecha. A la izquierda, bajo el glifo sostenido por Chac, el Dios de la Lluvia, parece flotar un textil. 


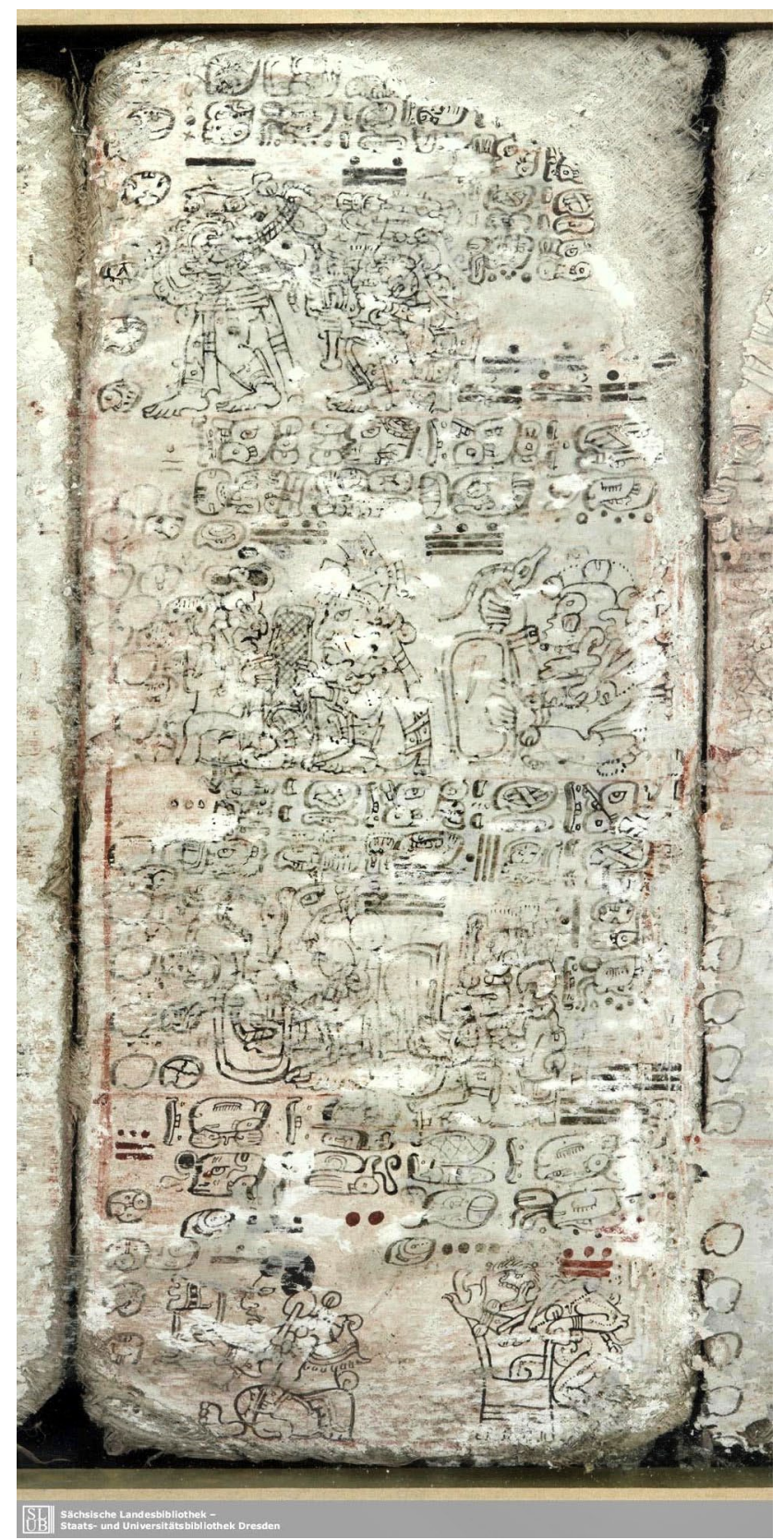

En el códice Trocortesiano o de Madrid, los marcos o cartuchos presentan dibujos y glifos asociados con fechas del calendario de 260 días, y sirven para determinar si un día particular realmente conviene para la realización de la actividad representada. De este modo, entre los pictogramas en los que el textil parece desempeñar algún papel, algunos revelan de manera evidente ofrendas depositadas sobre un textil, así como actividades relacionadas con el hilado, el tejido y la fertilidad.

Presentare pocos ejemplos dentro de los numerosos que encontré en el Trocortesiano porque esta informa-

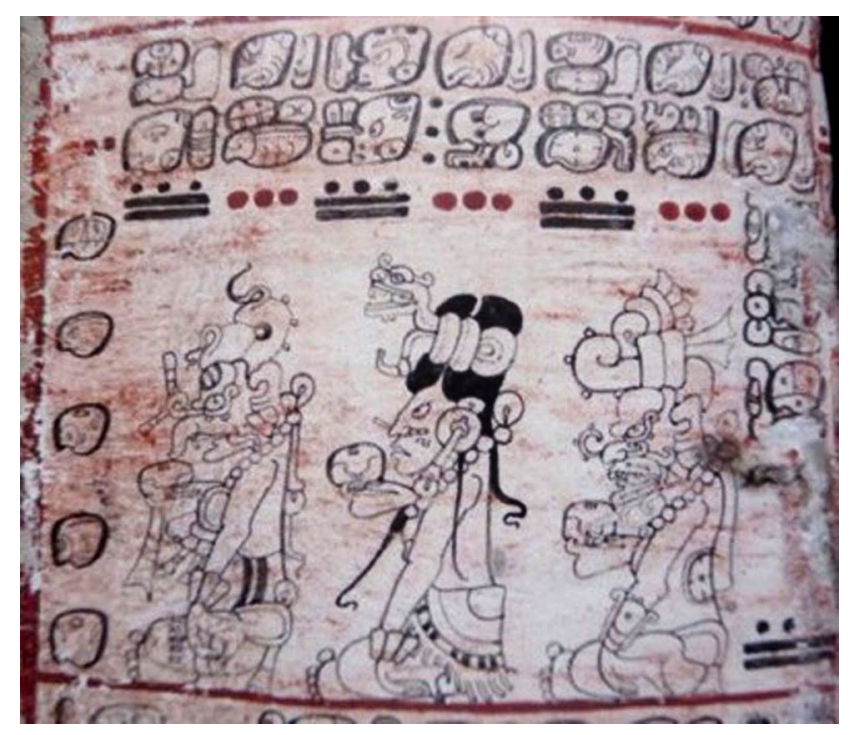

Foto 3: Códice Dresde, lámina 2 y lámina 22 b (C) Codex Dresdensis Mscr.Dresd.R.310

ción se encuentra en un artículo publicado en Anales del Museo de América en Madrid (Dupiech Cavaleri, 2016: XXIV, 169-210).

En la Lamina $74 b$, podemos observar tres divinidades, de izquierda a derecha, Itzamna, el Dios de la muerte y Chac, sosteniendo un textil y en la lamina $63 a$ (foto 4) notamos a la izquierda una divinidad femenina, Ixchel, que sostiene el glifo del maíz coronado de un brote de planta, y todo el conjunto parece reposar sobre un textil. El faldón de sus ropajes presenta una cenefa ornamentada con círculos que simbolizan el agua, lo que fortalece la asociación del textil con la fertilidad. A la derecha, Chac sostiene también el glifo del maíz, coronado por un grano de maíz, bajo el cual observamos un textil que ostenta el glifo del agua.

Los textiles eran parte de las ofrendas y tenían un valor sagrado indisoluble de la fertilidad y la cosmogonía. Hoy en día participan de las ceremonias y de los rituales. Pueden ser considerados como un remanente prehispánico, testimonio de una continuidad cultural, a la que fueron sumándose elementos exógenos a lo largo de los siglos.

\section{Venado, Perro y "S" acostada: unos ejemplos con ori- gen prehispánica.}

Las entrevistas con las bordadoras me permitieron establecer una nomenclatura de motivos recurrentes que fueron clasificados por temas: antropo-zoomorfo, 

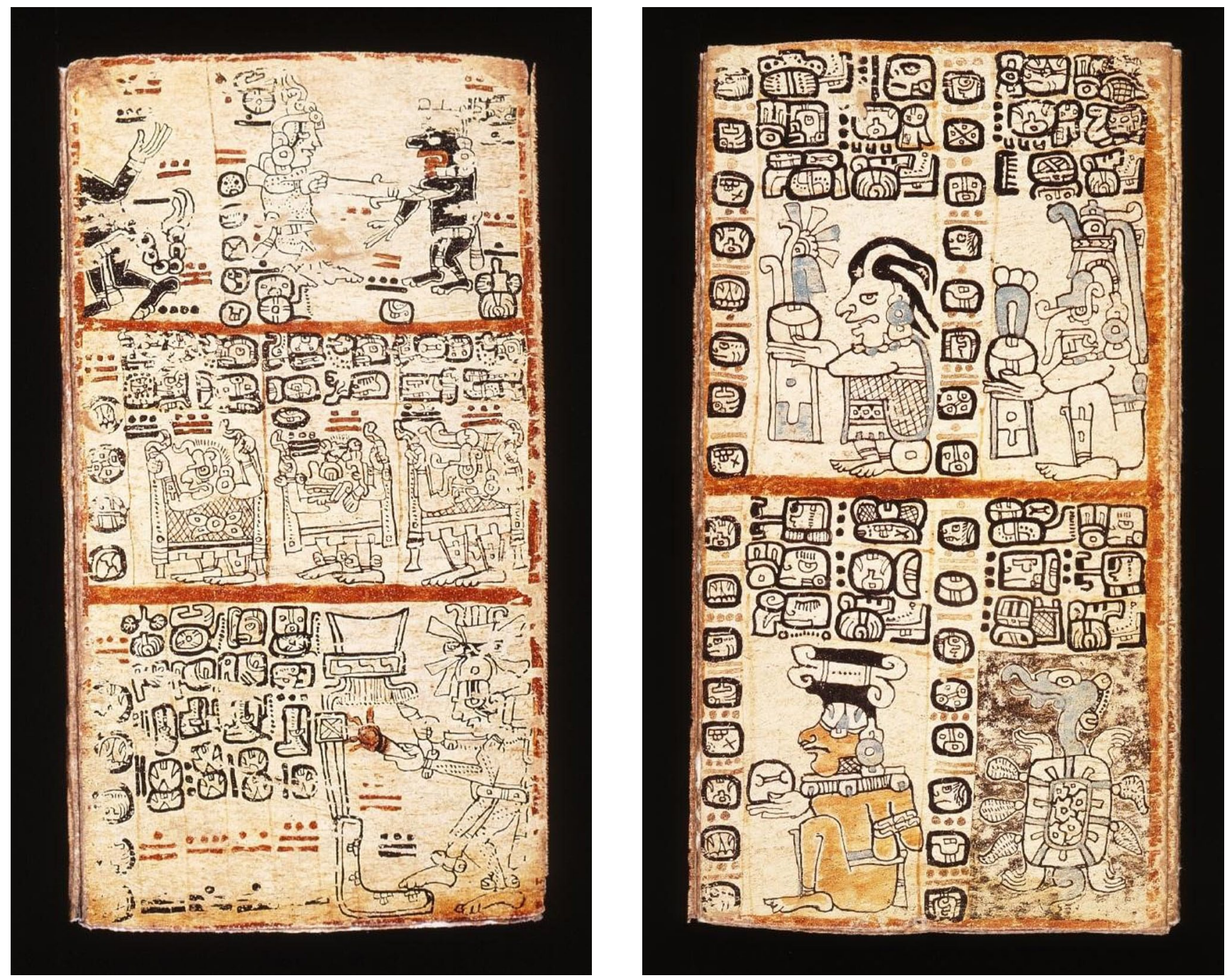

Foto 4: Códice Trocortesiano, lámina 74 b y lámina 63 a (C) Ministerio de Educación, Cultura y Deporte / NIPO: 551-09-052-7

zoomorfo, fitomorfo y geométrico. Algunos motivos tienen valor simbólico, otros carecen de él. La iconografía prehispánica, correlacionada gracias a los resultados de las excavaciones en sitios arqueológicos, nos informa sobre el papel de los animales en la vida religiosa de los mayas. Los cronistas españoles que describieron los usos y costumbres de los mayas al inicio de la Conquista citan entre las ofrendas que estos últimos tenían entre sus divinidades al jaguar, al venado, al perro, al pavo, al pez, a la iguana y a los insectos. Entre los motivos zoomorfos examinados con las bordadoras, observe la presencia recurrente de diez y nueve animales. El ser humano mesoamericano posee un alter ego animal o nahual que es atribuido a su nacimiento y le acompañará hasta la muerte. Los brujos, particularmente, tienen la facultad de transformarse temporalmente en animales bajo ciertas circunstancias. Esta capacidad se conoce en el centro de México bajo el nombre de nahualismo. En Yucatán, el brujo que toma en la noche la forma de un animal para atemorizar a las poblaciones es un uay-tan (Álvarez, 1984: vol. II, 309), un brujo que se transforma. Así, este se convertirá en uaay miztun (uay gato), en uay balam (uay jaguar), en uay cot (uay águila) etc... La mayoría de los motivos que bordaban las bordadoras mayas en los textiles transmitían hasta hace poco todo ese universo religioso.

El venado, Ceh, chan kee' (foto 5) es representado en los dechados de cinco bordadoras pero ya casi no lo bordan. El venado aparece en la cerámica prehispánica. En un 

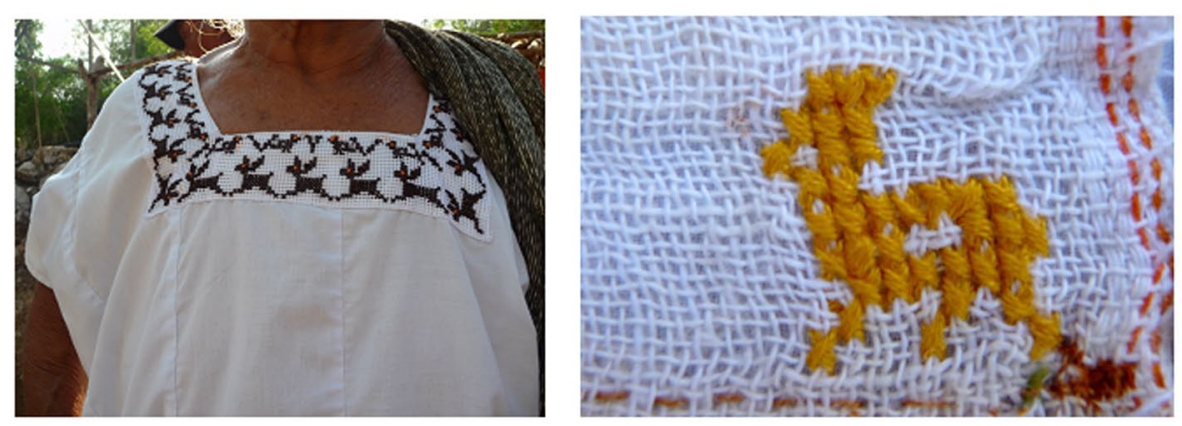
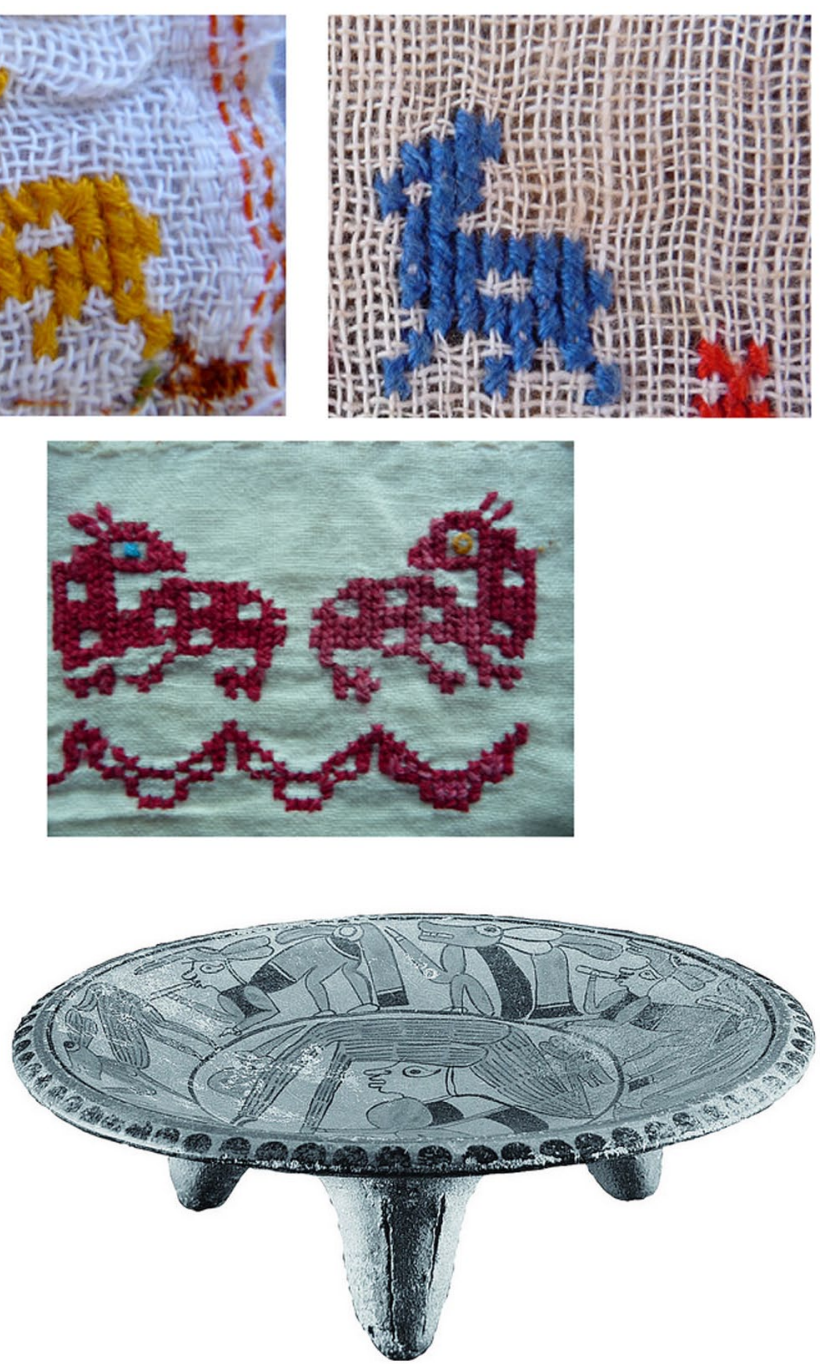

Foto 5: bordado de venado (ceh, chan kee') sobre dechados (C) D. Dupiech Cavaleri y venado pintado sobre cerámica prehispánica, Museo de Antropología, México y zona de Campeche, Museo Regional de Antropología, Palacio Canton, Mérida (C) dibujos fotográficos, Geovanni Martinez Guerra.

notamos en la lámina $107 a$ la representación de un pernil de venado encordelado, una ofrenda para Chac, el dios de la Lluvia (foto 6). La ofrenda de venado se menciona en el Libro de Chilam Balam (1984: 123) y en el Popol Vuh (Recinos, 1982) se cita al venado entre los primeros animales creados por los dioses. Los relatos que ilustran la relación entre el venado y el ser humano se transmiten por la tradición oral. Algunas viejas bordadoras siguen bordando el venado en sus hipiles. 


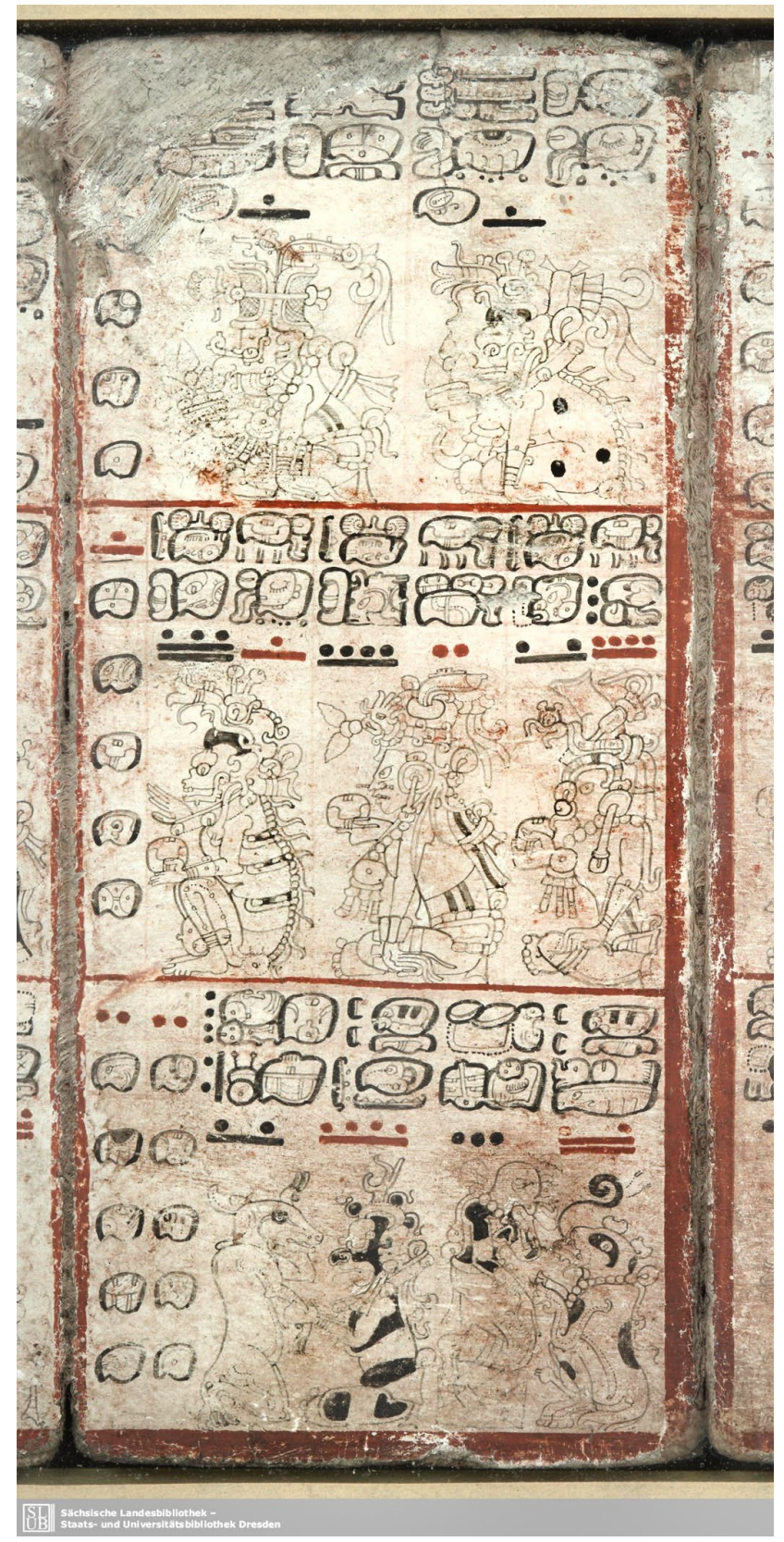

El perro (pek') es muy representado en los dechados de las viejas bordadoras. Dentro las entrevistadas, Adriana Napte Campos en Maní, es la más elocuente al respecto. Dice que «los campesinos ponían un hueso de perro en su sabucan o morral, como protección, cuando iban a trabajar a la milpa. El perro es protector, aleja las cosas malas, por eso es que las madres lo bordan en los hipiles de sus hijos, para protegerlos de lo malo que pudiera pasar». Adriana borda perros en los hipiles que ella viste y cuenta que siempre tiene perros en su casa para alejar al uay chivo. Los h-men o curanderos dicen que «el perro acompaña al alma de los muertos hacia el lugar de

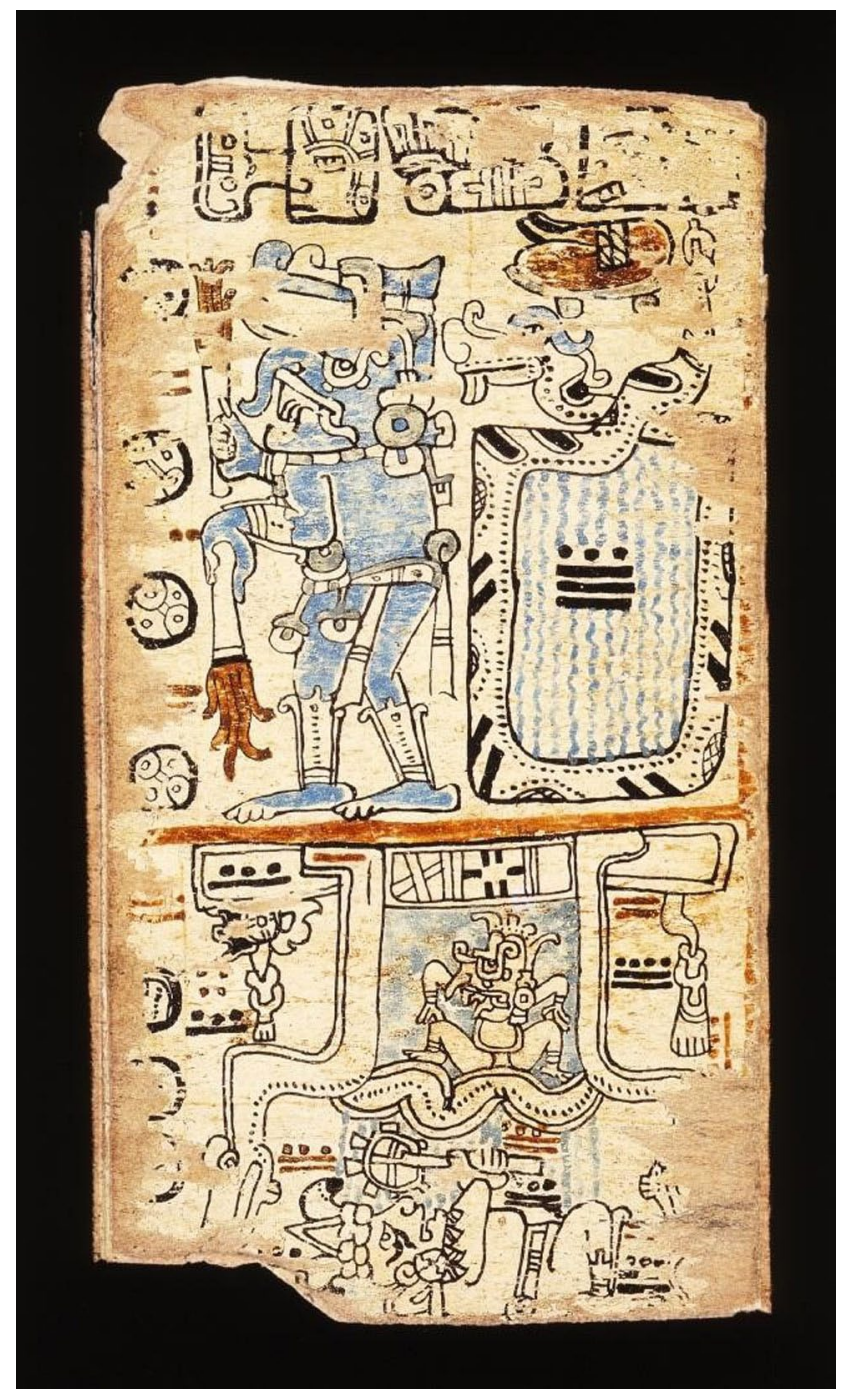

Foto 6: venado en el Códice Dresde, lámina 13c. (C) Codex Dresdensis-Mscr.Dresd.R.310 y ofrenda de pierna de venado en el Códice Trocortesiano, lámina 107a. (C) Ministerio de Educación, Cultura y Deporte / NIPO: 551-09-052-7.

descanso, nadie parte solo hacia su última morada, siempre va acompañado por el colibrí o el perro». En el diccionario de Motul (Álvarez, Vol. 1: 326) existen veinte y tres palabras que se refieren al perro. Por su parte, Diego de Landa evoca en múltiples ocasiones los sacrificios de perros cuyo corazón era ofrendado a las divinidades.

En los códices mayas, el perro se cuenta entre los animales más representados. En el códice de Madrid, lamina 78 c (foto 7), se puede ver al perro doméstico sobre una tortuga, al lado de un mono y un búho encaramado sobre el glifo de la muerte. Al perro se le asocia aquí con la muerte, con el inframundo y en el códice de Dresde, 
Foto 7: perros encontrados en los dechados de las bordadoras (C) D. Dupiech Cavaleri y ejemplos de representaciones del perro, Códice Trocortesiano, lámina 78c, (C) Ministerio de Educación, Cultura y Deporte /NIPO: 551-09-052-7 y Códice Dresde, lámina 30a. (C) Codex DresdensisMscr.Dresd.R.310.
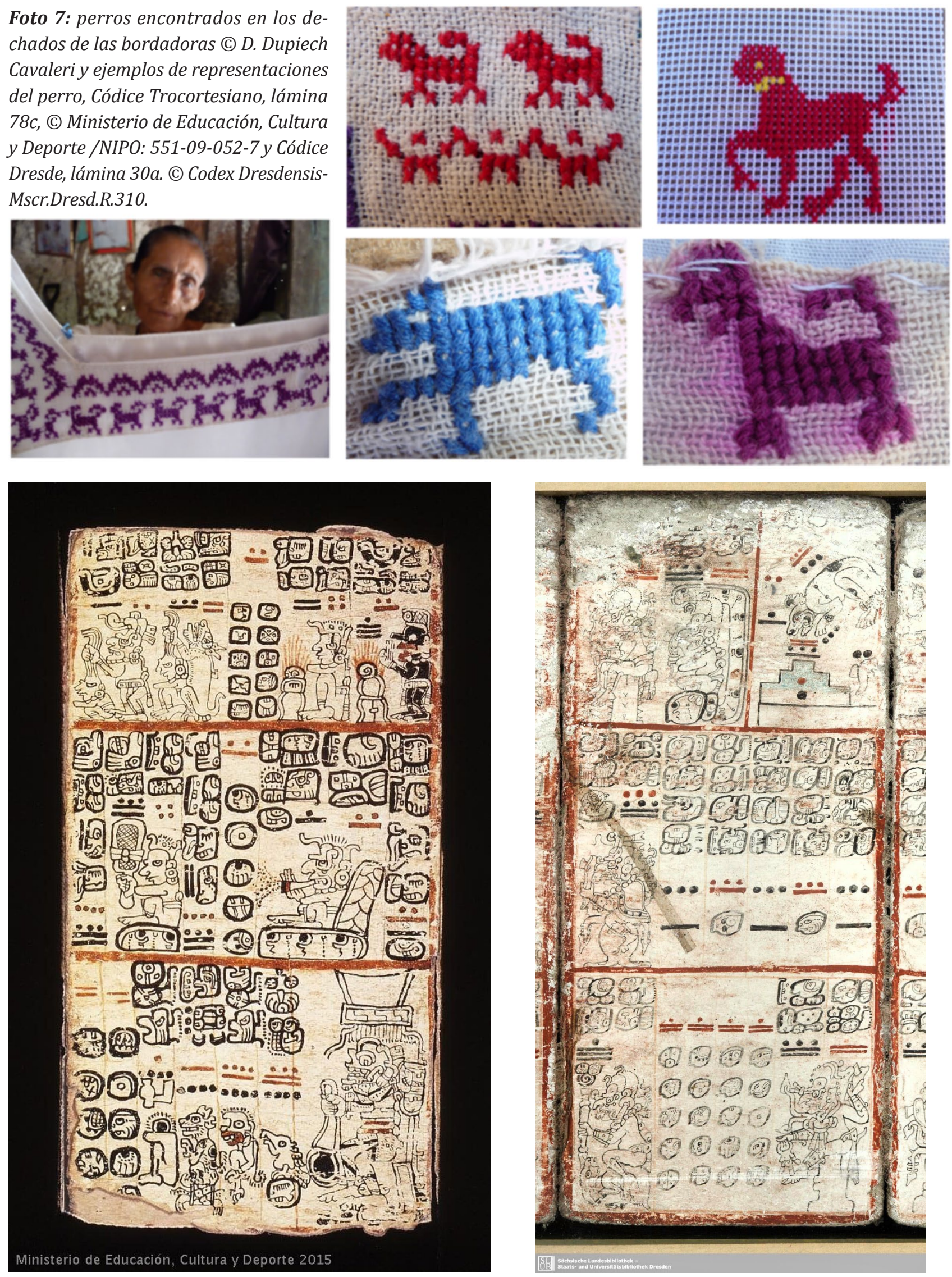

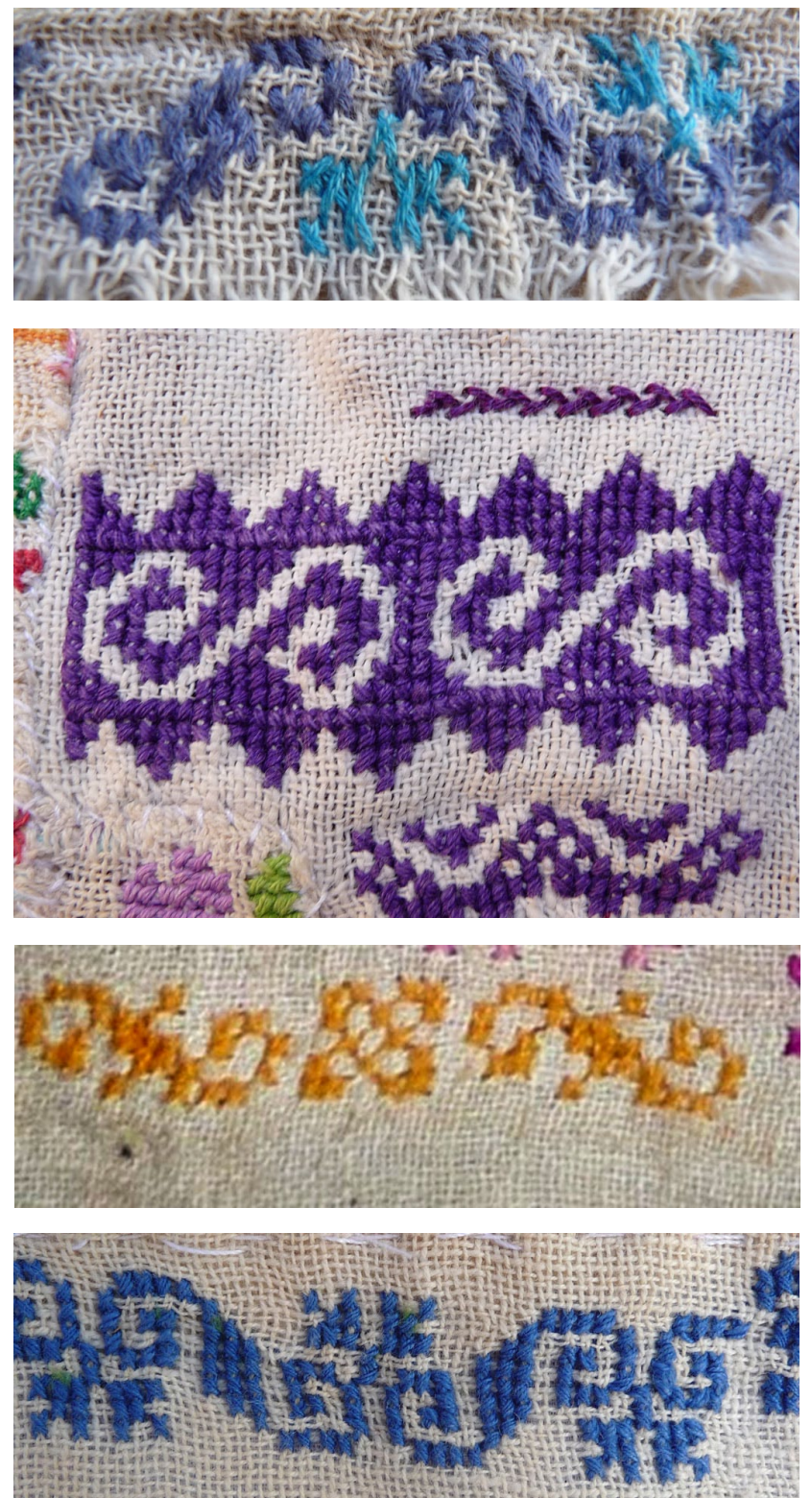

lámina $30 \mathrm{a}$, al perro doméstico con las patas atadas con una cuerda, se le representa listo para el sacrificio (foto 7). Es símbolo de fertilidad cuando se le sacrifica para que su sangre sea ofrendada a las divinidades. En el códice de Madrid, lámina 15 d, un cánido acompaña al dios Chac, el Dios de la lluvia en Yucatán. Está sembrando granos de maíz. Con relación al maíz también, en el mismo códice, lámina 18 d, el perro con manchas negras es representado con el glifo del maíz dentro del ojo y, abajo, ese mismo glifo aparece cerca de su mano. La lámina $19 b$ muestra un cánido a los pies de Chac, con el hocico apuntando hacia el glifo del maíz y, en la lámina 19 c, ante el dios del maíz.

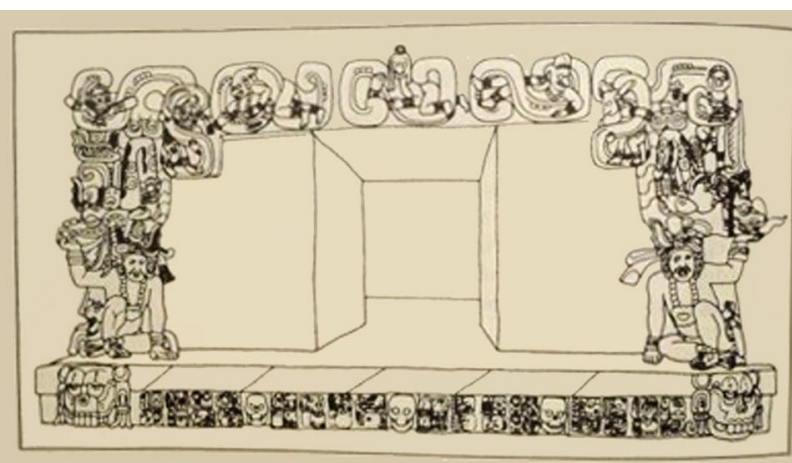

Ficura in.21. Puerta interior del Templo 22

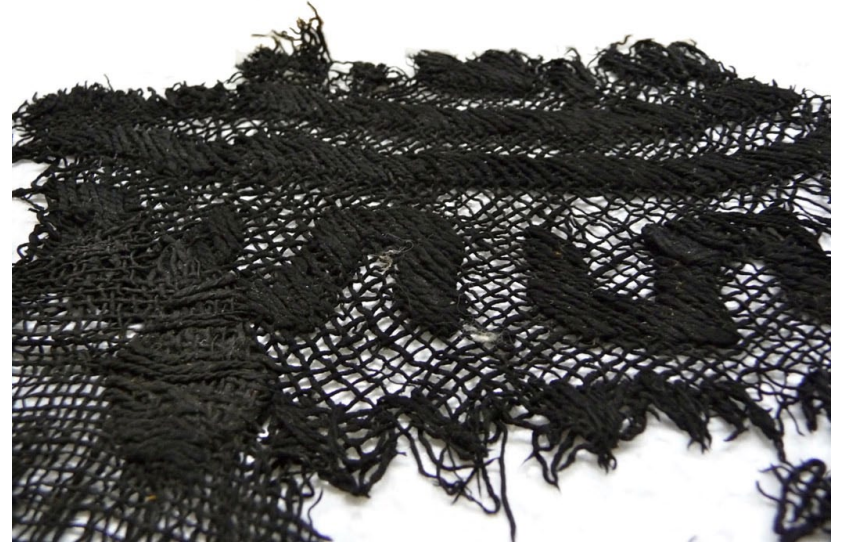

Foto 8: "S" bordado sobre dechados; "S" en la puerta interior del Templo 22, Copán, Honduras; « $S$ » en un fragmento de textil, cenote de Chichén Itzá, Museo Regional de Antropología, Palacio Cantón, Mérida (INAH), (C) dibujos fotográficos, Geovanni Martinez Guerra; Arriba y a la izquierda, dos motivos de "S" acostada, Códice Dresde, lámina 68a, (c) Codex Dresdensis-Mscr.Dresd.R.310; A la derecha, " $S$ " acostada sobre la cabeza de la diosa $O$ en lámina 21a, Códice Trocortesiano. (C) Ministerio de Educación.

Si bien el perro ya no es sacrificado ni consumido, sigue sin embargo asociado a la fertilidad y al maíz ya que supuestamente tiene que proteger a los campesinos cuando van a la milpa y porque las viejas bordadoras siguen ostentándolo en sus hipiles.

"S" acostada: encontré el motivo de "S" acostada en los dechados de seis bordadoras pero solo Matea Ku Kanche en Xocen lo borda en sus hipiles. J. Eric Thompson (1976: 248) nombra la "S" acostada Glifo 632 que identificó en varios monumentos en Yaxchilán, Quirigua, Naranjo y Piedras Negras. En Copán, Honduras, la "S" adorna un monumento donde volutas en forma de " $\mathrm{S}$ ", sostenidas en su base por bakabob (bacab), portadores de la dirección este 
y oeste, y que señala la trayectoria del sol, componen la parte superior de la puerta interior del Templo 22. Según David Friedel (1999: 148-149), estas volutas son nubes (foto 8). La "S" acostada aparece seis veces en el códice de Madrid y cuatro veces en el códice de Dresde (THOMPSON, 1976: 248). En particular, dos motivos de "S" acostada son visibles en el códice de Dresde, lámina 68 a, uno está ubicado arriba de dos Chac, sentados espalda con espalda, y el otro, al mismo nivel, aparece arriba de un chorro de agua (foto 8). En la lámina 21a del códice de Madrid, una "S" acostada y dos madejas de algodón forman el tocado de la diosa O, Chac Chel, la diosa hacedora de la lluvia, que aparece representada con una vasija de la que emanan chorros de agua (foto 8). Siguiendo con el códice de Madrid, en la lámina 60 b se puede observar a la diosa Chac Chel o diosa 0, con un tocado en forma de "S" acostada, que sostiene en sus manos el glifo del maíz y lámina $79 \mathrm{~d}$ la divinidad lleva también un tocado en forma de "S" acostada. (Weitlaner Johnson, 1976) describe un motivo de estrella y de hourglass shape (forma de reloj de arena) asociado a un motivo de "S" a la que designa como una espiral curvilínea en un rebozo proveniente de Sucilá, en Espita, Yucatán, que data de los años 1910-1920. La "S" acostada simboliza las nubes, muyal, munyal ou munial que traen la lluvia y fertilizan la tierra. Es probable que Matea Ku Kanche siga bordando este motivo, dado que aún se invoca en Xocén a divinidades relacionadas con las nubes anunciadoras de lluvia, compañeras de los Chacs (Terran y Ramussen, 2008, p. 231). El hecho de que hayan desaparecido de los textiles de las demás comunidades tiende a comprobar que el simbolismo que transmitían ya no se expresa de la misma manera.

Las mujeres siguen bordando motivos geométricos evocando a la serpiente tal como el pach kan o espalda de la serpiente y el $x$-manikte', que significa sin fin, eternidad. Esta puntada es asociada a un canto, $x$-manikben, que se le canta a la bordadora de $x$-manikte' cuando muere, al momento que el k'i'inam, el principio vital, se separa del cuerpo (Dupiech Cavaleri, 2017: 277- 299).

La desaparición del brocado en Yucatán no terminó de manera definitiva con una forma de escritura, sino que dio lugar a su transposición en otro tipo de soporte. La presencia de dibujos relacionados con la cosmogonía maya, relativos a conceptos de origen prehispánico, tiende a probar que los motivos elaborados por las bordadoras entrevistadas tienen un sentido. Hasta hace poco, las bordadoras mayas de Maní, de Xaya, de Xocén y de Xohuayán seguían bordando en los textiles los símbolos de su universo cosmogónico.

\section{Bibliografia}

ÁLVAREZ, Cristina, 1980, Diccionario etnolingüístico del idioma maya yucateco colonial, Volumen I: Mundo Fisico I, U.N.A.M., México.

Códice Trocortesiano, Museo de América, Madrid.

Codex Dresdensis - https://digital.slub-dresden.de/ kollektionssuche/, Saxon State and University Library, Dresden.

BARRERA VÁSQUEZ, Alfredo, Director, 2007, Diccionario Maya, Editorial Porrúa, México.

BARRERA VÁSQUEZ, Alfredo y Silvia RENDÓN, 1983, El libro de los libros de Chilam Balam, Traducido de sus textos por, basada en el studio, cotejo y recontrucción hechos por el primero, con introducciónes y notas. Fondo de Cultura Económic, México.

DUPIECH CAVALERI, Danielle, 2016, Los textiles mayas contemporáneos de Yucatán en el espejo del códice Trocortesiano, Anales del Museo de América, XXIV, 169-2010, https://sede.educacion.gob.es/ publiventa/anales-del-museo-de-america xxiv2016/ america-antropologia-museos/21079C

2017, Tradition textile maya du Yucatán (Mexique XXIème siècle). Usages rituels et codes symboliques, L'Harmattan, Paris.

FREIDEL David, Linda SCHELE y Joy PARKER, 1999, Centrando el mundo. El ombiligo del mundo, El cosmos Maya, tres mil años de la senda de los chamanes, Fundo de Cultura Económica, México.

LANDA, Fray Diego de, 2003, Relación de las cosas de Yucatán, Estudio preliminar, cronología y revisión del texto, María del Carmen León Cázares, CONACULTA/Cien de México.

MALHER LOTHROP Joy, 1992, Artifacts from the Cenote of Chen Itza, edited by Clemency Chase Coggins, Peabody Museum of Archeology \& Ethnology. Harvard University, Cambridge, Massachusetts.

MOREHART Christopher T., Jaime J. AWE, Michael J. MIRO, Vanessa A. OWEN, and Christophe G. HELMKE, June 2004, Ancient Textiles Remains From Barton Creek Cave, Cayo District, Belize, Mexicon, Vol. XXVI.

Popol Vuh, 1982, Las antiguas historias del Quiché, Traducidas del texto original con introducción y notas por Adrián RECINOS, Fondo de Cultura Económica.

QUEZADA, Sergio, 2010, Historia breve de Yucatán, Fondo de Cultura Económica, México.

Relaciones histórico-geográficas de la Gobernación de Yucatán, Mérida, Valladolid y Tabasco, 1983, Tomo I, Edición por Mercedes de la GARZA, Ana Luisa IZQUIERDO, María del Carmen León, UNAM, México.

SCHELE Linda \& GRUBE Nikolai, 1997, The Proceeding Of The Maya Hieroglyphic Workshop. The Dresden Codex, University of Texas at Austin, Transcribed and edited by Phil Wanyerka, March 8-9. 
SELER, Eduard, 2008, Las Imagenes de Animales en los Manuscritos Mexicanos y Mayas, Traducción Joachim von Mentz, Edición y estudios preliminar Brigida von Mentz, Segunda Edición, Casa Juan Pablos, México.

TERRAN Silvia y RAMUSSEN Christian H., 2008, Jinetes del cielo maya. Dioses de la lluvia, ediciones de la Universidad Autónoma de Yucatán, Mérida.
THOMPSON, J. Eric S., 1976, A Catalog of Maya Hierolyphs, University of Oklahoma Press, Third printing.

WEITLANER JOHNSON, Irmgard, 1976, Design Motifs on Mexican Indian Textiles, Vol. 1/II, Akademische Druck-uVerlagsanstalt, Graz, Austria. 